\title{
EFFECT OF MTA ON TWO TYPES OF FURCAL PERFORATION IN PRI- MARY MOLARS : A PROSPECTIVE CLINICAL TRIAL
}

\author{
Hamdy Mahmoud Badreldin ${ }^{1 *}$, Alaaeldin Abdallah Ismail ${ }^{2}$, Samy Yousef Elbayomy ${ }^{3}$
}

\begin{abstract}
Objective: Pulpotomy and pulpectomy usually are the most common techniques used in pulp therapy for primary teeth and despite the relative ease of performing this techniques iatrogenic errors may. Furcal perforation of common errors can be occurred during performing pulp therapy in primary molars. Two types of furcal perforation were selected according to time factor elapsed since their occurrence (recent and long standing) and these types of perforation treated with MTA. This study was directed to evaluate the ability of MTA to treat furcal perforation in primary molars regarding the time factor Subjects and Methods: thirty patients were selected 15 with accidently recently ( group A) furcally perforated molars and 15 with long standing old perforation (group B), and the two types sealed with MTA and then completed by conventional pulp therapy treatment either by pulpotomy or pulpectomy for group A, and pulpectomy for group B, then they followed up clinically and radiographically for 12 months. Result: there was statistically significant difference in success incidence between the two groups ( $\mathrm{p}=0.014)$; being higher in recent perforation. For long standing perforation; 10 patients out of 15 patients $(66.7 \%)$ showed success; while in recent perforation; 15 patients $(100 \%)$ showed success. Conclusion: recent type of perforation which repaired at the same visit has a higher success rate and better prognosis than old type or long standing in primary molars
\end{abstract}

KEY WORDS: Primary molars, furcal perforation, recent perforation, long standing perforation, MTA

\section{INTRODUCTION}

Root perforation is an artificial opening between the root canal system and the surrounding tissues of teeth, which may be created by the clinician error during entry into the root canal system and during cavity preparation or by resorption or caries, resulting in communication between root canal and periodontal tissue ${ }^{(1)}$.

Furcal perforation in primary teeth considered as an undesirable incident which affects the prognosis of the treatment, and any delay in repairing the furcal perforation results in the bacterial contamination of the perforation site which consequently leads to the gingival down-growth of the epithelium into the perforation area, inflammation, bone resorption, necrosis and eventual loss of the tooth ${ }^{(2,3)}$.

Treatment of root perforation can be achieved by surgical or non-surgical technique, however non-surgical which is more suitable for primary teeth involving uses of different materials. Such as material should be nontoxic, non-resorbable, radiopaque and bacteriostatic and should have good seal ability ${ }^{(2,4)}$. In addition, the material should present an osteogenic inductive capacity and

1. Assistant Lecturer, Pedodontics and Oral health, Faculty of Dental Medicine Al-Azhar University, Boys, Cairo

2. Assistant Professor, Pedodontics and Oral Health. Faculty of Dental Medicine, Al-Azhar University, Boys, Cairo

3. Professor, Pedodontics and Oral Health. Faculty of Dental Medicine Al-Azhar University, Boys, Cairo

-Corresponding author: badreldin_h@azhar.edu.eg

DOI: 10.21608/ajdsm.2020.42409.1116 
biocompatibility ${ }^{(4)}$. several materials were used in the perforation repair such as amalgam, calcium hydroxide, Intermediate restorative material (IRM), light-cured glass ionomer, gutta percha, calcium phosphate, Cavite, composite, Portland cement and MTA ${ }^{(5)}$. But there was no material have the whole ideal properties ${ }^{(1,5)}$.

Mineral tri oxide aggregate (MTA) was developed since 1993 by Mahmoud Torabinejad at Loma Linda University, it of consists of dicalcium silicate, tricalcium silicate, tricalcium aluminate, and tetracalcium, aluminoferrite. MTA come with many advantages such as antimicrobial action, biocompatibility, good sealing ability, low cytotoxicity, and ability to set in the presence of blood and moisture contamination. However, MTA still has many drawbacks as long setting time, and difficult handling characteristics ${ }^{(6)}$.

Many factors can determine the success rate of treatment of furcal perforation such as time factor which is important factor specially in primary teeth, as caries progress is faster than in permanent teeth and can reach the furcal area leaving pathologic furcal perforation in a short time ${ }^{(7)}$, the aim of this study directed to evaluate the effect of MTA in treatment of furcal perforation in primary molars regarding the time factor by comparison between two types of perforation ( old and recent).

\section{SUBJECTS AND METHODS}

Thirty patients were referred to or selected from the outside clinic of pedodontics Al-Azhar University-boys Cairo, after the patients were informed about the procedure and treatment, the parent signed an consent form.

Group A: recent perforation: This group included 15 patients having accidental furcal perforation by undergraduate students detected at the same clinic and sealed immediately at the same visit.

Group B : old standing perforation : 15 patient referred to the clinic with old perforation; its cause may be accidentally by other dentist or undergraduate student and not repaired at the same visit or due to caries.

Inclusion criteria included primary molars with iatrogenic furcal perforation and with sufficient coronal tooth structure to be restored. Exclusion criteria included: teeth with insufficient coronal structure or with insufficient periodontal support; molars with large periapical lesion including the succedenous tooth germ or with root resorption exceeding one third of the root length; very large perforation extending more than $2 / 3$ of pulpal floor area.

\section{Treatment protocol}

Tooth preparation: (1) Cleaning the work area, and ensured that rubber dam in the right place. (2)Irrigation and flushing the pulp chamber and perforation site with copious saline solution. (3) A moist cotton pellet of $2.5 \%$ Sodium Hypochlorite $(\mathrm{SH})$ was placed in the perforation site to control bleeding and disinfect perforation, then reassessment for the tooth was done regarding the pulp condition. a series of large, sterile cotton pellets were placed into the chamber with pressure to obtain temporary haemostasis. (4) After reassessment clinically and radiographically the teeth in group 1 was treated either by pulpotomy or pulpectomy and group II by pulpectomy. According to the current status and condition of the tooth if there were excessive continuous bleeding after 5 minutes of hemostasis indicating inflammation or in case of necrotic pulp tissues the tooth directed to pulpectomy otherwise it was treated by pulpotomy. Teeth in group B treated with pulpectomy

\section{Perforation treatment}

MTA mixing:- Neo MTA(NuSmile Ltd, Houston, USA) was used and the powder is mixed with sterile water in a ratio of 3:1 (powder to liquid) with a metal spatula on a glass slab to form the consistency of putty like paste. The material was transferred to the cavity by MTA carrier and condensed slightly to the site of perforation until complete covering of the 
perforation. Teeth were restored with Glass Ionomer or composite material. Periapical radiographs were taken to ensure that MTA glass ionomer filling (Fuji Equia, GC Corporation, Japan) and stainless steel crown are in the right place.

Pulpotomy procedures: After treatment of perforation the rest of the pulpal floor covered by the same material of perforation treatment, in $3 \mathrm{~mm}$ thickness by a cotton pellet damped with sterile water and squeezed well. The rest of the cavity filled with glass ionomer filling material. The tooth directed to fully restored with glass ionomer filling (Fuji Equia, GC Corporation, japan ) then preparation of stainless steel crown.

Pulpectomy procedures: this done for all molars in group B as well as some cases in group A. The working length determines by placing initial files in the canals then determining the length with periapical radiograph ( the length should be within 1-3 $\mathrm{mm}$ from the radiographic apex). Complete debridement of the pulpal tissue in the canal was done by headstrom file. Rotary endodntic files ( AF Baby Rotary, Fanta, China) system were used to complete the preparation. Irrigation was done by $\mathrm{SH}$ 2.5\%. Calcium hydroxide with iodoform (Metapex, Meta biomed. Korea) was used to obturate the prepared canals. The cavity floor coated with same material used in perforation to ensure the adequate sealing of the floor and perforation site. The final restoration was glass ionomer filling with glass ionomer filling (Fuji Equia, GC Corporation) then preparation of stainless steel crown. All patients were scheduled to be examined periodically every three months to assess the following: Tenderness to palpation, presences of swelling or fistula and pathological mobility.

Tooth was considered clinically successful when there is no sign of existence of any of the previous criteria and the tooth was functionally active

Radiographic evaluation: All the patient scheduled for radiographic examination with at
6,12 , months after treatment and case considered failure if on the following detected:

Presence of new Periapical, interradicular radiolucency, pathologic root resorption and If there was existing lesion under the bifurcation area and increase in size.

Data management and statistical analysis: were performed using the Statistical Package for Social Sciences (SPSS) version. 25. differences were analyzed with chi square $\left(\mathrm{c}^{2}\right)$ and fisher exact test, all $\mathrm{p}$-values are two-sided. $\mathrm{P}$-values $\leq 0.05$ were considered significant

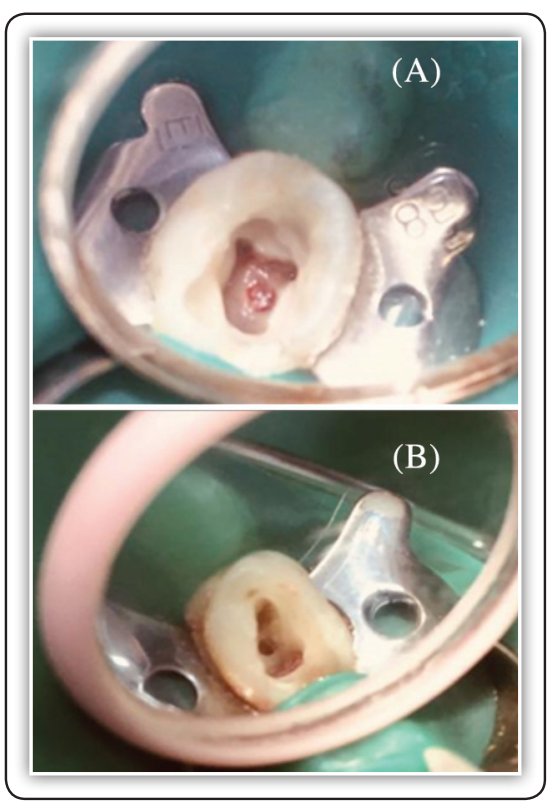

FIG (1) Two types of furcal perforation. a) Old standing perforation. b) Recent perforation

\section{RESULTS}

At 6 months, there was statistically significant difference in success incidence between the two groups ( $\mathrm{p}=0.014)$; being higher in recent perforation . For long standing perforation; 10 patients out of 15 patients $(66.7 \%)$ showed success; while in recent perforation; 15 patients (100\%) showed success. At 12 months, there was also statistically significant difference in success incidence between the two groups ( $\mathrm{p}<0.001)$; being higher in recent perforation. 
For long standing perforation; 6 patients out of 15 patients $(40.0 \%)$ showed success; while in recent perforation; 15 patients (100\%) showed success.

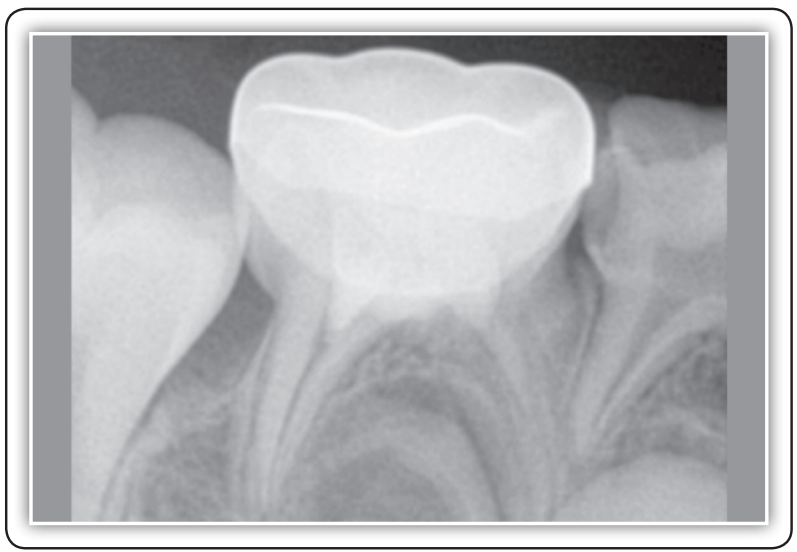

FIG (2) Lower second primary molar with furcal perforation after 12 months follow up in group A.

TABLE (1): Frequencies (n), percentages and results of Chi-square (x2) and fisher exact tests for comparison of success rate between the two perforation types in MTA

\begin{tabular}{|c|c|c|c|c|c|c|}
\hline \multicolumn{2}{|c|}{} & \multicolumn{2}{|c|}{ Group B } & \multicolumn{2}{c|}{ Group A } & \\
\cline { 3 - 7 } \multicolumn{2}{|c|}{} & Count & $\%$ & Count & $\%$ & \multirow{2}{*}{ P value } \\
\hline \multirow{2}{*}{$6 \mathrm{~m}$} & Failure & 5 & 33.3 & 0 & 0.0 & \multirow{2}{*}{0.014} \\
\cline { 2 - 6 } & Success & 10 & 66.7 & 15 & 100.0 & \\
\hline \multirow{2}{*}{$12 \mathrm{~m}$} & Failure & 9 & 60.0 & 0 & 0.0 & \multirow{2}{*}{$<0.001$} \\
\cline { 2 - 6 } & Success & 6 & 40.0 & 15 & 100.0 & \\
\hline
\end{tabular}

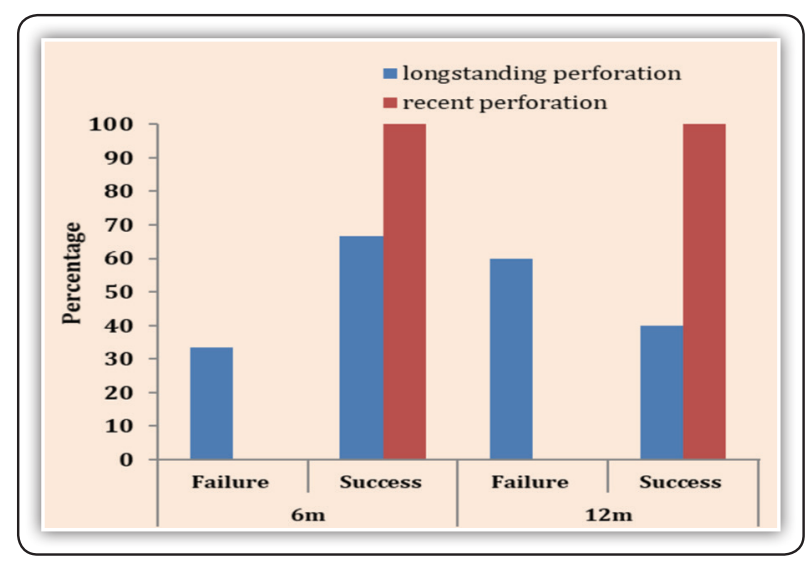

FIG (3) Bar chart representing in two groups of MTA at different point times in longstanding and recent perforation groups

\section{DISCUSSION}

This study compared 30 molars with furcal perforations divided into two groups according to the time factor into recent and old standing perforations. The result showed significant differences between the two groups in favor to the recent perforation. This highly success results regarding the recent perforation was attributed to the immediate sealing of the perforations in which the possibility of contamination is reduced, also the antibacterial properties of MTA can overcome the possibility of the slight bacterial contamination which my result during immediate repair ${ }^{(3,6)}$. the recent perforation showed low success rate.

Many trials come in favor with this current result in which they test the perforation treatment in relation to time such as studies done by Lantz \& Persson ${ }^{\left({ }^{89}\right)}$ which they produced root perforations in dogs that were treated either immediately or after some delay, and the results was most when perforations were sealed immediately. Moreover Seltzer et al ${ }^{(10)}$ treated 22 perforations in monkeys at intervals from immediately to 10 months postperforation. While the periodontium was damaged in all teeth, the most severe tissue destruction was in the untreated perforations and in teeth where treatment was delayed. The low success rate in the old standing group was mainly due to possible bacterial contamination, occurred along the period till treatment the perforation, the contamination may leads to the gingival down-growth of the epithelium into the perforation area, inflammation, bone resorption, necrosis and eventual loss of the tooth, however the furcation area in primary teeth exhibits certain different characteristics. The thin pulpal floor, higher prevalence of accessory canals, and wider dentin tubules found in primary teeth result with high permeability of pulpal floor ${ }^{(11)}$, and these factors are generally considered to cause furcation lesions in primary teeth with irreversible pulpitis or necrotic pulps. The longer the period the perforation left without treatment, the higher 
bacterial contamination and increase in the bacterial load and that can explain the higher failure rate in the old perforation group.

\section{CONCLUSION}

Recently treated perforation with MTA in primary molar has a better prognosis than old long standing perforation and has a higher success rate.

\section{REFERENCES}

1. Katge FA, Shivasharan PR, Patil D. Sealing ability of mineral trioxide aggregate Plus ${ }^{\mathrm{TM}}$ and Biodentine ${ }^{\mathrm{TM}}$ for repair of furcal perforation in primary molars: An in vitro study. Contemp Clin Dent. 2016; 7: 487-92.

2. Han L., Kodama S, Okii T. Evaluation of calcium-releasing and apatite-forming abilities of fast-setting calcium silicate-based endodontic materials. Inter endod J ,2015; 48 (2) 124-30.

3. Silveira CMM, Sánchez-Ayala A, Lagravère MO, Pilatti GL, Comes OMM. Repair of furcal perforation with mineral trioxide aggregate: long-term follow-up of 2 cases. J Can Dent Assoc. 2008;74(8):729.

4. Samuel A, Asokan S, P. R. Geetha, and Thomas S. Evaluation of sealing ability of Biodentine ${ }^{\mathrm{TM}}$ and mineral trioxide aggregate in primary molars using scanning electron microscope: A randomized controlled in vitro trial. Contemp Clin Dent. 2016; 7(3): 322-25.

5. Haghgoo R, Arfa S, Asgary S. Microleakage of CEM cement andProRoot MTA as furcal perforation repair materials in primaryteeth. Iran Endod J. 2013;8:187-90.

6. Hosoya N, Takigawa T, Horie T, et al. A review of the literature on the efficacy of mineral trioxide aggregate in conservative dentistry. Dent Mater J. 2019;38(5):693-700

7. Guedes RS, Piovesan C, Floriano I, et al. Risk of initial and moderate caries lesions in primary teeth to progress to dentine cavitation: a 2-year cohort study. Int J Paediatr Dent. 2016;26(2):116-24.

8. Lantz B, Persson PA. Experimental root perforation in dogs' teeth. A roentgen study. Odontol Revy. 1965: 16: 238-257.

9. Lantz B, Persson PA. Periodontal tissue reactions after root perforations in dogs' teeth. A histologic study. Odontol Tidskr. 1967: 75: 209-237.

10. Seltzer S, Sinai I, August D. Periodontal effects of root perforations before and during endodontic procedures. J Dent Res. 1970: 49: 332-339.

11. Arikan V, Sonmez H, Sari S. Comparison of Two Base Materials Regarding Their Effect on Root Canal Treatment Success in Primary Molars with Furcation 